\title{
RNA and actinomycin-D enhancement of learning in the carp'
}

STANLEY BATKIN, KAISER MEDICAL CENTER AND UNIVERSITY OF HAWAII WILLIAM T. WOODARD ${ }^{2}$, ROBERT E. COLE AND JOHN B. HALL, UNIVERSITY OF HAWAII

A total of 172 carp were tested in three experiments on a T-maze visual discrimination task. As compared to saline controls, carp given intracranial injections of either RNA or actinomycin-D made significantly more correct responses on a $24-\mathrm{hr}$. test. This effect may relate to the increased avai!ability of nucleotides.

Several investigators have reported enhancement of learning or memory following administration of ribonucleic acid (RNA) or of nucleic acid and protein synthesis stimulators (Cook, Davidson, Davis, Green, \& Fellows, 1963; Chamberlain, Rothschild, \& Gerard, 1963). Others have noted impairment of performance with antimetabolites (Davis \& Agranoff, 1966). The purpose of this study was to directly compare the effect of RNA and antimetabolites using a single species and technique.

\section{Method}

A total of 172 Japanese carp (Cyprinus carpio) -7 to $9 \mathrm{~cm}$ in fork length-were tested in groups of 12 or 16 fish each. Fish were trained on a visual discrimination task in a $\mathrm{T}$-maze. This involved the avoidance of a Plexiglas blocking door on which there was a single mid-line blue stripe. Reward consisted of escape from the narrow confines (alley width $=4 \mathrm{~cm}$ ) and shallow water of the maze $(23 \mathrm{~mm}$ deep) to an open area $(114 \mathrm{~cm}$ square) and deeper water ( $37 \mathrm{~mm}$ deep). Three separate experiments were conducted. Each experiment consisted of three training sessions of 40 trials each. The intertrial interval was $2.5 \mathrm{~min}$. The second session was run $24 \mathrm{hr}$. after the first, the third either 11 days (Experiments I and II) or 15 days (Experiment III) after the second. The experimenter, different for each experiment, was blind to the chemical treatment.

The groups tested, and their respective treatments in Experiments I to III, were as follows: (a) RNA (IP): RNA, $2.0 \mathrm{mg}, 100 \mathrm{ul}$, intraperitoneally (IP), Experiment I; (b) SAL (IP): Saline, 0.14 N, 100 ul, Experiment I; (c) RNA (IC): RNA, $0.2 \mathrm{mg}, 10 \mathrm{ul}$, intracranially (IC), Experiments I, II, and III; (d) PUR (IC): puromycin, 90.0 ug, $10 \mathrm{ul}, \mathrm{IC}$, Experiment I; (e) ACT (IC): actinomycin-D, $1.5 \mathrm{ug}, 10 \mathrm{ul}, \mathrm{IC}$, Experiments I and III; (f) SAL (IC): Saline, 0.14 N, $10 \mathrm{ul}$, IC, Experiments I, II, and III; (g) NOR: normal (not injected), Experiments I and III. The IP injections were continued for 21 days, with Session 1 on the ninth day of injection, Session 2 on the tenth day, and Session 3 on the twenty-first day. The IC injections were given only on Sessions 1 and 2. All injections were given $1 \mathrm{hr}$. prior to the training sessions. The IC injections were placed just anterior to the forebrain using a needle fitted with a guard allowing a penetration of $2 \mathrm{~mm}$. Tricaine methanesolfonate (MS222-Sandoz), $3 \mathrm{mg}$ in $200 \mathrm{cc}$ water, was used to sedate the fish for the IC injections in Experiments I and II, but not III.

Resuits

The measure of performance for each fish was the total number of correct responses on each session. Since the effects of certain chemicals have been tested in more than one experiment, the data from these replications have been pooled in order to show results of greater generality. This data is presented in Fig. 1. Mann-Whitney $U$ tests performed on the pooled data revealed no significant effects on Session 1. On Session 2 there were significant differences between RNA (IC) and SAL (IC) $(p<.001), \operatorname{ACT}$ (IC) and SAL (IC) $(p<$ $.001)$, RNA (IC) and NOR ( $<<.01)$, and NOR and SAL (IC) $(p<.05)$. On Session 3 there was a significant difference between NOR and SAL (IC) $(p<.05)$. No other differences were significant $(p>.05)$.

Similar statistical tests were performed on individual experiments. The difference between RNA (IC) and SAL (IC) were consistently significant across all three experiments. In the two experiments involving ACT (IC) and SAL (IC) the results were also consistent. Differences involving the pooled NOR data were not consistent across individual experiments.

The data from the RNA (IP) and PUR (IC) groups indicated no significant effects $(p>.05)$ on any of the training sessions. No conclusions will be drawn from this data since these experiments were not repeated.

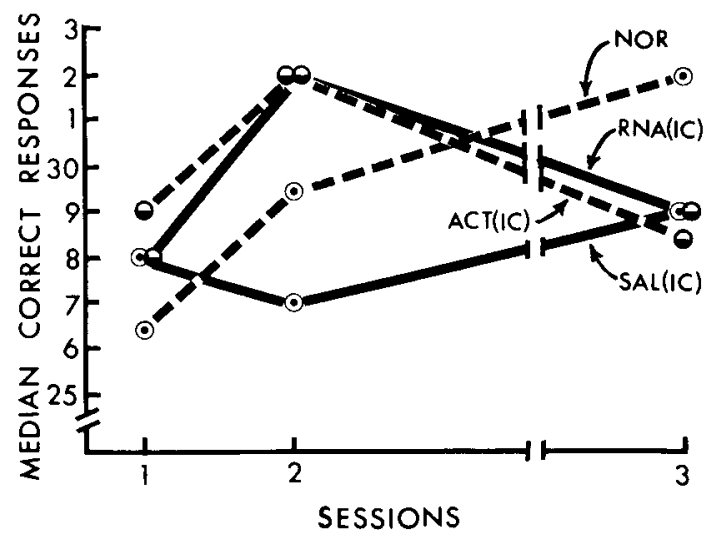

Fig. 1. Median number of correct responses given on each training session. Based on pooled data from all replications. 


\section{Discussion}

Barondes \& Jarvik (1964) found that IC-injected actinomycin-D did not interfere with learning and memory although it caused a marked inhibition of DNA-dependent RNA synthesis in mice. This inhibition would prevent the use of nucleotides for DNA-dependent RNA synthesis and thus might allow their utilization in other processes necessary for learning. Such an increased availability of nucleotides may play a role in the enhancement effect of actinomycin-D noted in the present study. This relationship between RNA metabolism and learning has been suggested by the studies of Cook et al (1963), Chamberlain et al (1963), and Glasky \& Simon (1966) with Plotnikoff (1966) who reported enhancement of learned responses from increased levels of nucleic acid precursors or nucleic acid synthesis.

The lack of an enhancement effect in Barondes \& Jarvik's experiment may be due to their use of extremely short retention periods ( 1 to $3 \mathrm{hr}$.). This might not have allowed sufficient performance decrement in the control groups for an enhancement effect to be apparent.

Since both the RNA and actinomycin-D injected fish showed enhanced performance, the common denominator may relate to increased availability of nucleotides.

If the beneficial effect on performance resulted from the increased availability of nucleic acid substrates, additional studies using specific nucleotides should be of interest. Such studies are now in progress.

\section{References}

Barondes, S. H., \& Jarvik, M. E. The influence of actinomycin-D on brain RNA synthesis and on memory. $J$. Neurochem., 1964, 11, 187-195.

Chamberlain, T. J., Rothschild, G. H., \& Gerard, R. W. Drugs affecting RNA and learning. Proc. Natl. Acad. Sci., 1963, 49. 918-924.

Cook, L., Davidson, A. B., Davis, D. J., Green, H., \& Fellows, E. J. Ribonucleic acid: effect on conditioned behavior in rats. Science, 1963, 141, 268-269.

Davis, R. E., \& Agranoff, B. W. Stages of memory formation in goldfish: evidence for an environmental trigger. Proc. Natl. Acad. Sci., 1966, 55, 555-559.

Glasky, A. J., \& Simon, L. N. Magnesium pemoline: enhancement of brain RNA Polymerases. Science, 1966, 151, 702-703.

Plotnikoff, N. Magnesium pemoline: enhancement of learning and memory of a conditioned avoidance response. Science, 1966 , 151, 703-704.

\section{Notes}

1. We gratefully acknowledge the encouragement of Professor Terence A. Rogers, University of Hawaii. Professor Louis Di Carlo, Syracuse University, and Robert Morris assisted in the original stages of the study. Puromycin dihydrochloride and RNA, reagent grade, were purchased from Nutritional Biochemicals Corporation. Actinomycin-D was donated by Merck, Sharp \& Dohme. 2. Now at the Department of Psychology, Dalhousie University. 3. The present results were reported at The American Association for the Advancement of Sc ience Berkeley Meeting, December, 1965. 